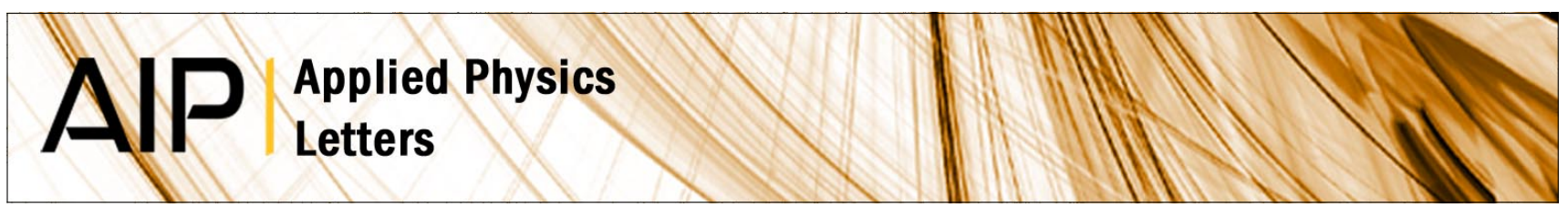

\title{
Tunnel magnetoresistance in
}

\section{La0.7Ca0.3MnO3/PrBa2Cu3O7/La0.7Ca0.3MnO3}

Z. Sefrioui, V. Cros, A. Barthélémy, V. Peña, C. León et al.

Citation: Appl. Phys. Lett. 88, 022512 (2006); doi: 10.1063/1.2162674

View online: http://dx.doi.org/10.1063/1.2162674

View Table of Contents: http://apl.aip.org/resource/1/APPLAB/v88/i2

Published by the American Institute of Physics.

\section{Additional information on Appl. Phys. Lett.}

Journal Homepage: http://apl.aip.org/

Journal Information: http://apl.aip.org/about/about_the_journal

Top downloads: http://apl.aip.org/features/most_downloaded

Information for Authors: http://apl.aip.org/authors

\section{ADVERTISEMENT}

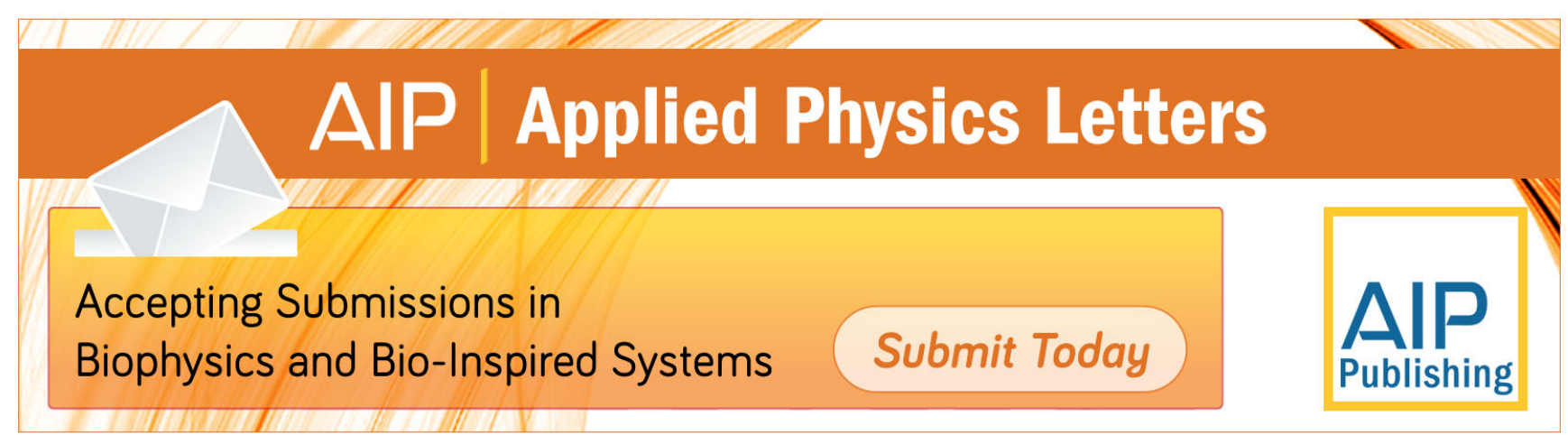




\title{
Tunnel magnetoresistance in $\mathrm{La}_{0.7} \mathrm{Ca}_{0.3} \mathrm{MnO}_{3} / \mathrm{PrBa}_{2} \mathrm{Cu}_{3} \mathrm{O}_{7} / \mathrm{La}_{0.7} \mathrm{Ca}_{0.3} \mathrm{MnO}_{3}$
}

\author{
Z. Sefrioui, ${ }^{\text {a) }}$ V. Cros, and A. Barthélémy \\ Unité mixte de Physique CNRS/Thales, route départementale 128, 91767 Palaiseau, France \\ V. Peña, C. León, and J. Santamaria \\ GFMC. Departamento Fisica Aplicada III, Universidad Complutense de Madrid, 28040 Madrid, Spain \\ M. Varela and S. J. Pennycook \\ Condensed Matter Sciences Division, Oak Ridge National Laboratory, Oak Ridge, Tennessee 37831-6031
}

(Received 2 August 2005; accepted 15 November 2005; published online 13 January 2006)

We report large tunneling magnetoresistance in $\mathrm{La}_{0.7} \mathrm{Ca}_{0.3} \mathrm{MnO}_{3}(8 \mathrm{~nm}) / \mathrm{PrBa}_{2} \mathrm{Cu}_{3} \mathrm{O}_{7}(2.4 \mathrm{~nm}) /$ $\mathrm{La}_{0.7} \mathrm{Ca}_{0.3} \mathrm{MnO}_{3}(50 \mathrm{~nm})$ junctions. The coherent growth of the cuprate on the manganite allows the deposition of ultrathin barriers which are continuous and flat over long lateral distances. Epitaxial strain causes the top layer to be a weaker ferromagnet without a significant decrease in the spin polarization. (C) 2006 American Institute of Physics. [DOI: 10.1063/1.2162674]

Magnetic tunnel junctions (MTJs) based on complex manganese oxides have recently been the focus of much interest due to their potential applications in spintronics and magnetic recording. ${ }^{1-6}$ The high degree of spin polarization of the conduction band of manganites, confirmed for $\mathrm{La}_{0.7} \mathrm{Sr}_{0.3} \mathrm{MnO}_{3}$ (LSMO) by photoemission spectroscopy, ${ }^{7}$ is expected to yield large tunneling magnetoresistance (TMR). Indeed, transport experiments leading to large degrees of spin polarization seem to confirm this expectation $[P=95 \%$ at $4 \mathrm{~K}^{3}$ and $86 \%$ at $77 \mathrm{~K}$ (see Ref. 8)]. However, while large values of TMR have been obtained in experiments done at low temperature, a rapid decrease of TMR has been often observed in manganite-based tunnel junctions with increasing temperature, vanishing at temperatures below the Curie temperature of the bulk electrodes. ${ }^{4,8-11}$ It has been suggested ${ }^{4}$ that the rapid drop of TMR with temperature might be due to a weakened ferromagnetism resulting from structural distortions or modified chemical bonding at the interface. In addition, epitaxial mismatch strain might also influence the intrinsic tendency of manganites to phase separation modifying the magnetic properties of the electrodes at the interface. ${ }^{12}$ In fact, this might provide an explanation for the different values of TMR reported for LSMO-based tunnel junctions with different insulating barriers. ${ }^{3,6}$ These problems suggest that further research is necessary to examine the influence of interface properties on the TMR.

In this letter, we report on MTJs with manganite electrodes and a new barrier of $\mathrm{PrBa}_{2} \mathrm{Cu}_{3} \mathrm{O}_{7}(\mathrm{PBCO})$. We show that the chemically coherent growth of $\mathrm{PBCO}$ on $\mathrm{La}_{0.7} \mathrm{Ca}_{0.3} \mathrm{MnO}_{3}$ (LCMO) allows depositing nanometer thick barriers continuous and atomically flat over large lateral distances, yielding MTJs with large TMR. Furthermore, we show that the anisotropic mismatch strain with the LCMO induces a depressed $T_{\mathrm{C}}$ and enhanced coercivity in the top layer allowing the independent magnetization switching of both LCMO layers. This result constitutes additional evidence of the importance of chemical bonding and interface properties on the spin polarization and TMR of MTJs.

\footnotetext{
${ }^{\text {a) }}$ Author to whom correspondence should be addressed; on leave from: Universidad Complutense de Madrid, Spain; electronic mail: sefrioui@fis.ucm.es
}

Films were grown on (100) $\mathrm{SrTiO}_{3}$ (STO) substrate in a high pressure $(3.4 \mathrm{mbar})$ pure oxygen sputtering system at high temperatures $\left(900{ }^{\circ} \mathrm{C}\right)$. This technique provides a very thermalized and ordered growth at a slow rate $(1 \mathrm{~nm} / \mathrm{min})$ that allows an accurate control of the layer thickness. Bottom electrode was grown thicker $(50 \mathrm{~nm})$ than the top electrode $(8 \mathrm{~nm})$ to favor different coercivities of both electrodes. Junctions were patterned using optical lithography and Ar ion milling. Further details on the patterning process can be found elsewhere. ${ }^{3,4}$ Magnetic measurements were performed by superconducting quantum interference device (SQUID; Quantum Design) magnetometry. Electrical measurements were performed in the current-perpendicular-to-plane geometry using four-terminal dc method with the magnetic field applied parallel to the in-plane direction of the samples. Electron microscopy observations were carried out in a VG Microscopes HB501UX dedicated scanning transmission electron microscope (STEM).

Figure 1(a) shows a cross-sectional TEM image of the sample showing that the layers are continuous and flat over long lateral distances. Figure 1(b) displays an atomic resolution Z-contrast image of the PBCO layer (marked with a black arrow) in between the LCMO electrodes. The interfaces are sharp and coherent, and the barrier structural quality is very high. The Z-contrast image shows that the interface plane $\mathrm{Cu}-\mathrm{O}$ chains are lacking that is, the LCMO termination is a $\mathrm{MnO}_{2}$ plane, and the $\mathrm{PBCO}$ layer growth starts in a $\mathrm{BaO}$ plane. The $\mathrm{CuO}$ chains plane can be seen at
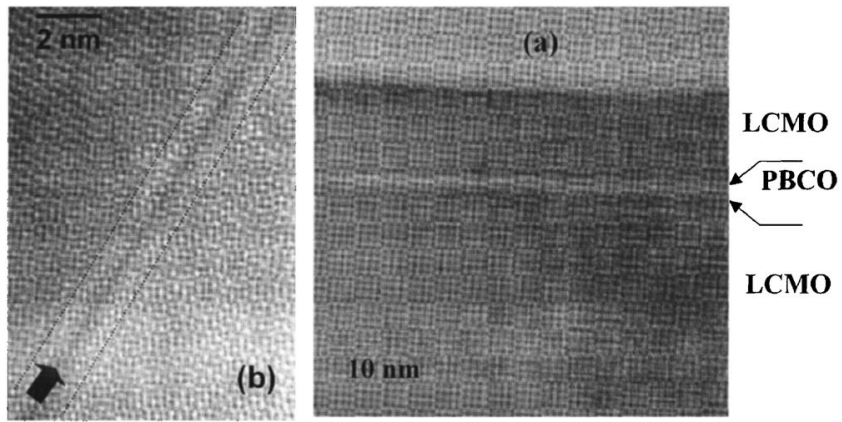

FIG. 1. (a) Cross-sectional TEM image of LCMO/PBCO/LCMO trilayer. (b) Z-contrast STEM low-magnification image of the same sample. 


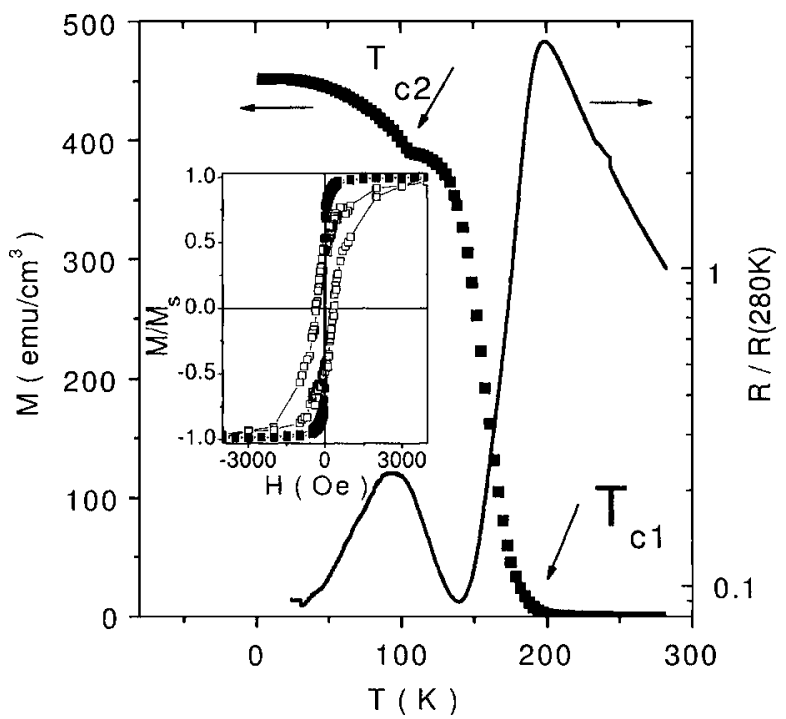

FIG. 2. Magnetization curve vs temperature for LCMO $(8 \mathrm{~nm}) / \mathrm{PBCO}$ $(2.4 \mathrm{~nm}) / \mathrm{LCMO}(50 \mathrm{~nm})$ trilayer (square symbol) and electrical resistance vs temperature for LCMO $(8 \mathrm{~nm}) / \mathrm{PBCO}(2.4 \mathrm{~nm}) / \mathrm{LCMO}(50 \mathrm{~nm})$ junction (line symbol). Inset: Hysteresis loops measured at $5 \mathrm{~K}$ of single LCMO $(50 \mathrm{~nm})$ film directly grown on STO substrate (dark symbol) and of LCMO $(8 \mathrm{~nm})$ film grown on 2 u.c. PBCO buffer on STO (open symbol).

the center of the 2 unit cell (u.c.) thick barrier layer with the darkest contrast, due to their reduced atomic density as compared to other planes of the structure. On Fig. 2, we show the temperature dependence of the magnetization in the LCMO $(8 \mathrm{~nm}) / \mathrm{PBCO}(2.4 \mathrm{~nm}) / \mathrm{LCMO}(50 \mathrm{~nm})$ trilayer (square symbols). It is clear from the Fig. 2 that the magnetization curve shows two different Curie temperatures corresponding to the bottom $\left(T_{\mathrm{C} 1} \sim 200 \mathrm{~K}\right)$ and top $\left(T_{\mathrm{C} 2} \sim 108 \mathrm{~K}\right)$ electrodes. The same Curie temperatures have been observed (not shown) in single LCMO layers directly grown on STO and on PBCO buffer of the same thickness as in the trilayers. Figure 2 also shows a resistance versus temperature curve of the tunnel junction, evidencing the metal-insulator (MI) transition that occurs at both Curie temperatures. The $T_{\mathrm{C}}$ value for the $8 \mathrm{~nm}$ film is in good agreement with the one reported by Bibes et al. ${ }^{13}$ for thin LCMO films on $\mathrm{SrTiO}_{3}$. Such reduction for the $8 \mathrm{~nm}$ film could be attributed to a large tensile strain between PBCO and LCMO. In plane lattice parameters of PBCO are $a=0.3865 \mathrm{~nm}$ and $b=0.3931 \mathrm{~nm}$. Therefore, there is nearly perfect lattice matching with the manganite along $a$ direction, while $\left(b_{\mathrm{PBCO}}-b_{\mathrm{LCMO}}\right) / b_{\mathrm{LCMO}}$ $\sim 1.58 \%$ along the $b$ direction. The reduced Curie temperature has been attributed to the weakening of the doubleexchange mechanism due to strain-related charge localization at the interface. ${ }^{13}$ In order to examine the coercive field of both electrodes, we have measured the magnetization loops after zero field cooling. In the inset of Fig. 2, we show the hysteresis loops measured at $5 \mathrm{~K}$ of single LCMO $(50 \mathrm{~nm})$ film directly grown on STO substrate (dark symbols) and of LCMO $(8 \mathrm{~nm})$ film grown on 2 u.c. PBCO buffer on STO (open symbols) with a magnetic field applied parallel to the plane direction of the samples. The thicker LCMO $(50 \mathrm{~nm})$ film exhibits a small coercive field $H_{\mathrm{C} 1}$ $\sim 30$ Oe, while the $8 \mathrm{~nm}$ film grown on $\mathrm{PBCO}$ shows a significantly enhanced value of $H_{\mathrm{C} 2} \sim 380 \mathrm{Oe}$. The reduced $\mathrm{Cu}-$ rie temperature and enhanced coercivity of the top layer may be related to changes of the electronic structure at the interface caused by strain, to modified chemical bonding, or even

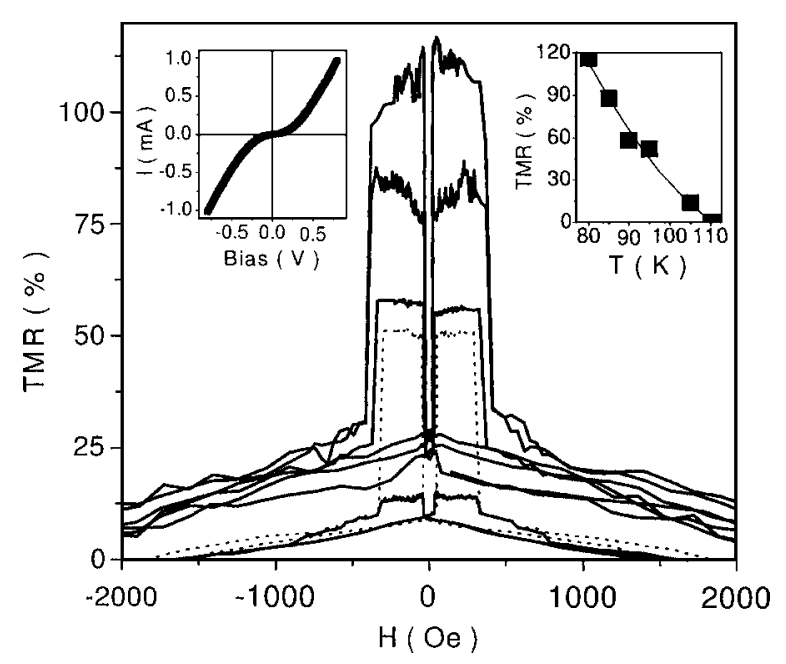

FIG. 3. TMR vs magnetic field for LCMO $(8 \mathrm{~nm}) / \mathrm{PBCO}(2.4 \mathrm{~nm}) / \mathrm{LCMO}$ $(50 \mathrm{~nm})$ junction measured at temperatures ranging from 80 (top) to $105 \mathrm{~K}$ (bottom). Inset: TMR versus temperature (right) and $I-V$ characteristic of the junction (left). The solid line is a guide for the eye.

to charge transfer as recently reported for cuprate/manganite heterostructures. ${ }^{14}$ Interface effects are expected to be more pronounced in the thinner top layer than in the bottom one. The difference in coercive fields ensures antiparallel alignment of the magnetization of bottom and top electrodes over a wide field interval.

In Fig. 3, we display the TMR measured at a bias voltage of $V_{\mathrm{dc}}=10 \mathrm{mV}$ as a function of magnetic field for LCMO $(8 \mathrm{~nm}) / \mathrm{PBCO}(2.4 \mathrm{~nm}) / \mathrm{LCMO}(50 \mathrm{~nm})$ junction measured at temperatures ranging from 80 to $110 \mathrm{~K}$. The temperature has been kept in this range to avoid complications due to the antiferromagnetic transition known to occur in the barrier material at lower temperatures. ${ }^{15}$ The junction area was 50 $\times 50(\mu \mathrm{m})^{2}$. TMR was defined as TMR $=\left(R_{\mathrm{ap}}-R_{\mathrm{p}}\right) / R_{\mathrm{p}}$, where $R_{\mathrm{ap}}$ and $R_{\mathrm{p}}$ are, respectively, the tunnel resistances in the antiparallel and parallel magnetization configurations. The junction resistance switches sharply from parallel to antiparallel state (and vice versa) at field values corresponding to the coercive fields obtained from SQUID measurements (see the inset of Fig. 2). The TMR ratio is about $110 \%$ at relatively high temperature $(T=80 \mathrm{~K})$ and then decreases with increasing temperature. In fact, it vanishes at the MI transition temperature of the top electrode (see Fig. 2). This result, together with the sharp switching between parallel and antiparallel alignment of the magnetization of both LCMO electrodes, reflects the high structural quality of our MTJs. Furthermore, the $I-V$ characteristic (see the inset of Fig. 3) shows a typical nonlinear and slightly asymmetric behavior generally observed in MTJs with different electrodes. All these observations indicate that our MTJs are free of pinholes and that tunneling is the dominant transport mechanism. ${ }^{16}$ Assuming that the polarization decreases almost as the magnetization, ${ }^{4}$ that is, the polarization of the thin layer at $80 \mathrm{~K}$ is $56 \%$ of its value at $5 \mathrm{~K}$ (see Fig. 2), whereas the polarization of the thick layer is nearly constant on the temperature range $5-80 \mathrm{~K}$, we can use Jullière formula, ${ }^{17}$ TMR $=2\left[\left(0.56 P_{0}\right) P_{0} /\left(1-P_{0}\left(0.56 P_{0}\right)\right)\right]$, to obtain a value of the spin polarization of at least $80 \%$ at $5 \mathrm{~K}$.

In summary, we have shown TMR in excess of $100 \%$ on LCMO $(8 \mathrm{~nm}) / \mathrm{PBCO}(2.4 \mathrm{~nm}) / \mathrm{LCMO}(50 \mathrm{~nm})$ junctions. Structural analysis has confirmed the high quality of our 
samples with atomic flat interfaces between both materials. Epitaxial mismatch strain at the interface causes depressed $T_{\mathrm{C}}$ and enhanced coercivity in the top LCMO layer, but does not decrease significantly the spin polarization of the tunneling current.

One of the authors (Z.S.) gratefully acknowledges financial support from Flores Valles. Work supported by MCYT MAT 2002-2642, CAM GR-MAT-0771/2004, UCM PR3/0412399, and Fundación Ramón Areces. This research was partially sponsored by the Laboratory Directed Research and Development Program of ORNL, managed by UT-Batelle, LLC, for the U.S. Department of Energy under Contract No. DE-AC05-00OR22725. The authors thank M. Bibes for fruitful discussion.

${ }^{1}$ J. M. De Teresa, A. Barthélémy, A. Fert, J.-P. Contour, F. Montaigne, and P. Sensor, Science 286, 507 (1999).

${ }^{2}$ J. M. De Teresa, A. Barthélémy, A. Fert, J. P. Contour, R. Lyonnet, F. Montaigne, P. Sensor, and A. Vaures, Phys. Rev. Lett. 82, 4288 (1999).

${ }^{3}$ M. Bowen, M. Bibes, A. Barthélémy, J.-P. Contour, A. Anane, Y. Lemaître, and A. Fert, Appl. Phys. Lett. 82, 233 (2003).

${ }^{4}$ V. Garcia, M. Bibes, A. Barthélémy, M. Bowen, E. Jacquet, J.-P. Contour, and A. Fert, Phys. Rev. B 69, 052403 (2004).
${ }^{5}$ M. Viret, M. Drouet, J. Nassar, J.-P. Contour, C. Fermon, and A. Fert, Europhys. Lett. 39, 545 (1997).

${ }^{6}$ Y. Lu, X. W. Li, G. Q. Gong, G. Xiao, A. Gupta, P. Lecoeur, J. Z. Sun, Y. Y. Wang, and V. P. Dravid, Phys. Rev. B 54, R8357 (1996).

${ }^{7}$ J. Park, E. Vescovo, H. J. Kim, C. Kwon, R. Ramesh, and T. Venkatesan, Nature (London) 392, 794 (1998).

${ }^{8}$ M. H. Jo, N. D. Mathur, N. K. Todd, and M. G. Blamire, Phys. Rev. B 61, R14905 (2000).

${ }^{9}$ T. Obata, T. Manako, Y. Shimakawa, and Y. Kubo, Appl. Phys. Lett. 74, 290 (1999).

${ }^{10}$ J. O’Donnel, J. N. Eckstein, and M. S. Rzchowski, Appl. Phys. Lett. 76, 218 (2000).

${ }^{11}$ J. S. Noh, T. K. Nath, C. Eom, J. Z. Sun, W. Tian, and X. Q. Pan, Appl. Phys. Lett. 79, 233 (2001).

${ }^{12}$ E. Dagotto, T. Hotta, and A. Moreo, Phys. Rep. 344, 1 (2001).

${ }^{13}$ M. Bibes, Ll. Balcells, S. Valencia, J. Fontcuberta, M. Wojcik, E. Jedryka, and S. Nadolski, Phys. Rev. Lett. 87, 067210 (2001).

${ }^{14}$ A. Hoffmann, S. G. E. te Velthuis, Z. Sefrioui, J. Santamaría, M. R. Fitzsimmons, S. Park, and Y. M. Varela, Phys. Rev. B 72, 140407(R) (2005).

${ }^{15}$ S. Uma, W. Schnell, E. Gmelin, G. Rangarajan, S. Skanthakumar, J. W. Lynn, R. Walter, T. Lorenz, B. Büchner, E. Walker, and A. Erb, J. Phys.: Condens. Matter 10, L33 (1998).

${ }^{16}$ J. J. Akerman, J. M. Slaughter, R. W. Dave, and I. K. Schuller, Appl. Phys. Lett. 79, 3104 (2001).

${ }^{17}$ M. Jullière, Phys. Lett. A 54, 225 (1975). 